\title{
Prospettive teoriche per lo studio della contaminazione tra ambienti di insegnamento/ apprendimento fisici e virtuali
}

\author{
Theoretical perspectives for the study of the \\ contamination between physical and virtual \\ teaching/learning environments
}

\section{Agnese Del Zozzo e George Santi}

Facoltà di Scienze della Formazione, Libera Università di Bolzano - Italia;

Nucleo di Ricerca Didattica della matematica, Università di Bologna - Italia

Sunto / Proponiamo un'analisi degli effetti della presenza delle tecnologie digitali sulle dinamiche di insegnamento/ apprendimento nelle pratiche didattiche nelle aule scolastiche. Descriviamo due episodi - uno scambio di messaggi nello Stream di Google Classroom e un estratto di una chat privata su Whatsapp tra due compagni di classe. Analizziamo tali episodi con una lente teorica composta da il "Triangolo della didattica” (Chevallard \& Joshua, 1982), e la prospettiva sociologica, proposta da D’Amore (2005), che considera la classe come società. Le tecnologie digitali abilitano nuovi e complessi ambienti di insegnamento/apprendimento il cui studio richiede una visione sistemico relazionale come quella offerta dal Triangolo. Inoltre, si mostra come tali ambienti possono essere considerati a loro volta delle società con le proprie pratiche costitutive e meta-pratiche extrafunzionali che sono in relazione con le società nelle aule scolastiche. Mostriamo come l'interazione tra queste prospettive teoriche permette di caratterizzare i processi di insegnamento/ apprendimento quando ambienti fisici e virtuali si contaminano a vicenda.

Parole chiave: Triangolo di Chevallard; classe virtuale; società; tecnologie digitali; pratiche e meta-pratiche.

\begin{abstract}
We propose an analysis of the effects on the teaching/learning practices of mathematics that stem from digital technologies in school classrooms. We describe two episodes - an exchange of messages in the Stream of Google Classroom and an extract from a private Whatsapp chat between two classmates. We analyze such episodes with a theoretical lens based on the Chevallard Triangle of Didactics (Chevallard \& Joshua, 1982) and the sociological perspective, proposed by D’Amore (2005), considering the classroom as a society. Digital technologies enable new complex teaching/learning environments whose study require a systemic and relational approach as the one offered by the concept of Triangle. Moreover, we show how such environments in turn can be considered societies with their constitutive practices and extra-functional meta-practices that are linked with the societies that originate in the school classrooms. We show how the interaction between these theoretical perspectives allows us to characterize teaching/learning processes when physical and virtual environments contaminate each other.
\end{abstract}

Keywords: Chevallard Triangle; virtual classroom; society; digital technologies; practices and meta-practices.

\section{1} Premessa

Per migliorare l'efficacia della nostra comunicazione e per non rischiare fraintendimenti, riteniamo necessario esplicitare alcuni elementi preliminari.

- Contesto. II nostro contesto socio-culturale di riferimento è quello italiano e, da un punto di vista istituzionale/scolastico, ci collochiamo al livello di scuola seconda- 
ria di secondo grado'. Questo significa che quando usiamo termini legati al mondo scolastico quali "classe, aula, legge" ci riferiamo al loro significato nel contesto suddetto, con la consapevolezza degli ovvi limiti di generalizzabilità.

- Terminologia. Per evitare che i rischi della metafora dell'oggetto denunciati da Sfard (2008) sfuggano al nostro controllo, riteniamo importante esplicitare ciò che intendiamo con l'espressione "tecnologie digitali" e caratterizzare il tipo di ambienti di insegnamento/apprendimento (I/A) cui ci riferiamo. Con I'espressione "tecnologie digitali" indicheremo qualunque combinazione realizzabile di hardware e software. Quindi, ad esempio, una persona che usa un browser su un PC, sta usando una specifica istanza di tecnologia digitale; un'aula con un PC collegato ad un proiettore con cui viene visualizzato un video è un ambiente in cui è presente una specifica istanza di tecnologia digitale.

Considereremo due diversi ambienti di I/A: fisico e virtuale.

L'ambiente di I/A fisico è circoscritto allo spazio fisico, dove uno o più individui con il ruolo di allievo e uno o più individui con il ruolo di insegnante interagiscono in una dinamica di I/A. Un esempio di tale ambiente è l'aula scolastica, luogo dove la classe, qui intesa come l'insieme degli studenti, partecipa ad una lezione dell'insegnante.

L'ambiente di I/A virtuale è limitato allo spazio virtuale e si crea quando uno o più individui con il ruolo di allievo e uno o più individui con il ruolo di insegnante interagiscono in una dinamica di I/A mediata da un'istanza di tecnologia digitale. Gli individui possono interagire ed entrare in relazione tra loro sia nel primo ambiente che nel secondo. Quando accade che gli stessi individui sono tra loro in relazione di I/A sia in un ambiente fisico che in un ambiente virtuale, emerge un nuovo contesto generato dalla relazione e dalla contaminazione dei primi due. Tuttavia, una volta chiariti tali contorni, dichiariamo che per non appesantire la lettura, talvolta ci serviremo di espressioni più brevi quali ad esempio classe fisica in riferimento al primo ambiente e classe virtuale in riferimento al secondo.

- Distinzione e relazione tra persone fisiche e account. Riteniamo importante chiarire che: gli individui in un ambiente fisico sono persone fisiche (PF); gli individui in un ambiente virtuale sono account (A). Inoltre, la corrispondenza tra le persone fisiche e gli account NON è necessariamente biunivoca. Infatti, possono verificarsi situazioni in cui: ad ogni persona fisica corrisponde uno ed un solo account $(\# P F=\# A)$, la stessa persona fisica gestisce più di un account $(\# P F<\# A)$ e lo stesso account è gestito e controllato da più persone fisiche (\#PF $>$ \#A). Naturalmente, in un contesto di I/A le persone fisiche possono operare un uso consapevole e strategico degli account per effettuare particolari operazioni che abbiano rilevanza didattica. Ad esempio: è possibile attuare diversi livelli di monitoraggio sfruttando i diversi livelli di autorizzazione alle azioni degli account; un'unica persona fisica può decidere di raffinare e separare i diversi ruoli assunti durante le proprie interazioni con gli altri individui del medesimo contesto usando account diversi, ciascuno specifico per ogni ruolo; ecc.

- Invito al dialogo. La complessità del tema trattato nel presente articolo e la vastità della letteratura che potrebbe essere rilevante per una più approfondita descrizione sono tali da rendere parzialità e incompletezza ineliminabili. Pertanto, consci di questo, non riteniamo il presente contributo un punto di arrivo ma un

1. La scuola secondaria di secondo grado in Italia dura cinque anni e corrisponde all'ultimo anno di scuola media e alla scuola media superiore o alle scuole professionali nel Canton Ticino. 
punto di partenza, uno stimolo che vorremmo poter arricchire con le impressioni, le riflessioni e le esperienze di chi lo leggerà. Quindi, in parallelo alla versione del contributo sulla rivista, avvieremo un canale di comunicazione con i lettori che invitiamo a comunicarci riflessioni, commenti, suggerimenti e critiche delle quali potremo tenere conto per creare un'appendice dinamica che arricchisca l'articolo originale. Tale canale è un form online creato e gestito dai due autori del presente lavoro ed è accessibile al seguente link: https://forms.gle/ uf3Rdg6EY9JauSzq5.

Il panorama scientifico della didattica della matematica, ad oggi, offre numerosi strumenti teorici che permettono di studiare e caratterizzare le dinamiche di insegnamento/apprendimento (I/A), le cui sfaccettature e modalità dipendono fortemente dallo specifico contesto in cui avvengono.

Le tecnologie digitali, quando introdotte in un sistema didattico, impattano su elementi portanti e basilari che spaziano dal piano strumentale a quello strategico, dal piano personale a quello sociale, dal piano pragmatico a quello affettivo.

Se si pensa alla tradizionale classe di matematica, i primi elementi a venire in mente sono l'aula scolastica in cui si svolge la lezione di matematica con l'insegnante e i suoi studenti. In Borba, Askar, Engelbrecht, Gadanidis, Llinares e Aguilar (2016) viene messo in evidenza come la tecnologia digitale abbia completamente destrutturato questa immagine, abilitando nuove tipologie di classi e di modalità di lavoro che, a loro volta, innescano differenti dinamiche di natura socio-culturale:

"Allo stato attuale, sembra chiaro che la tecnologia digitale sta "decostruendo" la nozione di aula. [...] La tecnologia mobile, la PLN², gli oggetti per l'apprendimento digitale e altri artefatti stanno "allargando" I'aula, trasformandola a tal punto che si riesce a malapena a riconoscerla come tale. [...] In questo scenario, I'aula regolare non funge più da locus per l'educazione. Poltrone, sedie e tavoli nella casa degli studenti, i bar e gli internet café sono le nuove aule. Le classi ribaltate cambiano la nozione di ciò che è dentro e fuori della classe e cambiano i ruoli degli studenti e degli insegnanti».

(Borba et al., 2016, pp. 605-606, traduzione degli autori)

Pertanto, inquadrare e descrivere con specificità e precisione su cosa e come agisce tale impatto assume particolare importanza e la ricerca in didattica della matematica in questo ambito sta raccogliendo, a livello internazionale, gli sforzi di numerosi ricercatori. Borba et al. (2016) delineano cinque diverse correnti di sviluppo di ricerca relative all'uso delle tecnologie digitali in didattica della matematica:

1. la prima riguarda I'uso di dispositivi mobili;

2. la seconda riguarda i MOOCs (Massive Online Open Courses) che sono corsi 
online aperti ad un numero qualunque di partecipanti ai quali non sono richiesti particolari prerequisiti;

3. la terza riguarda collezioni di materiali didattici e learning objects cioè «[...] qualunque entità, digitale o non digitale, che può essere usata per l'apprendimento, l'educazione o la formazione» (Risk, 2002, p. 6, traduzione degli autori);

4. la quarta riguarda I'uso delle tecnologie per l'apprendimento collaborativo;

5. la quinta riguarda corsi blended, che combinano esperienze di I/A online con quelle faccia-a-faccia, usati in ambito di formazione degli insegnanti.

Intendiamo fornire un quadro teorico per accogliere la sfida di Borba et al. (2016) ed interpretare la complessità dei nuovi spazi di I/A derivanti dall'interazione tra classi fisiche e classi virtuali. In particolare, ci focalizziamo sulle possibili caratterizzazioni che la relazione triadica insegnante-allievo-Sapere (i-a-S) può assumere e quali sono i suoi effetti sui processi di I/A. La prospettiva secondo la quale guardiamo le interazioni tra classi fisiche e virtuali è di tipo sistemico-relazionale per evidenziare come gli studenti attuano l'apprendimento e le condizioni che lo attivano o lo inibiscono. Un altro filone di analisi - di grande rilievo e complementare a quello scelto in questo studio - riguarda il ruolo che le tecnologie digitali possono assumere nel contribuire a delineare i tratti distintivi della cognizione e dell'apprendimento in matematica e i processi che li caratterizzano.

Il presente studio, che prende le mosse nell'ambito del progetto di ricerca VirMath della Libera Università di Bolzano e che può essere localizzato a cavallo tra il primo e il quarto punto dell'elenco precedente, si concentra a livello di scuola secondaria di secondo grado e si propone di analizzare un sistema didattico per I'I/A della matematica in cui ambienti fisici contaminano e sono contaminati da ambienti virtuali.

Drijvers, Ball, Barzel, Heid, Cao e Maschietto (2016) presentano un panorama delle ricerche sull'uso delle tecnologie digitali nel particolare caso dei livelli scolastici pre-universitari. Per quanto riguarda l'uso delle tecnologie per la comunicazione, gli Autori mettono in evidenza un'interessante distinzione tra la comunicazione attraverso la tecnologia digitale e la comunicazione della tecnologia digitale, nel senso della comunicazione dell'output tecnologico. La comunicazione attraverso la tecnologia digitale fa riferimento sia a tecnologie di visualizzazione, che permettono di esporre, proiettare o condividere idee matematiche, sia a tecnologie di condivisione e collaborazione, che permettono alle persone che le usano di scambiarsi idee e materiali.

Nel presente lavoro, ci proponiamo di analizzare due esempi di comunicazione matematica realizzata attraverso la tecnologia in due contesti diversi tra loro che pur nella loro specificità possono essere rappresentativi di due situazioni molto diffuse in un contesto di I/A arricchito dalle tecnologie: I'uso di una piattaforma didattica digitale e la comunicazione via chat privata tra studenti.

Nel par. 3 introdurremo alcuni strumenti teorici con i quali inquadreremo tali contesti di comunicazione e apprendimento caratterizzati e permessi dall'uso di tecnologie digitali. Nel par. 4 analizzeremo e interpreteremo i due esempi di implementazioni di contesti di apprendimento che prevedono I'uso di tecnologie digitali e mostreremo gli effetti derivanti dal loro uso sull'I/A della matematica. II par. 5 conclude il lavoro con delle riflessioni più generali e di natura teorica. 


\section{Quadro teorico}

Nell'obiettivo di accogliere adeguatamente la complessità del sistema didattico da noi considerato, il quadro teorico scelto si compone di due elementi:

- Considereremo dapprima modelli sistemici che permettono di inquadrare i soggetti e le relazioni che caratterizzano un contesto didattico. II classico Triangolo della didattica di Chevallard e Joshua (1982), che generalizza le situazioni di I/A in matematica, corredato dalla nozione di Tetraedro elaborato da Albano, Faggiano e Mammana (2013) che si riferisce allo specifico caso dell'e-learning (nel seguito: Tetraedro)

- Sfruttando la prospettiva sociologica di classe intesa come società proposta da D'Amore (2005), individueremo alcune variabili di struttura del particolare contesto didattico che stiamo esaminando e che emergono come rilevanti.

\subsection{Modelli sistemici delle relazioni didattiche}

Chevallard e Joshua (1982) inquadrano in quello che viene chiamato il Triangolo della didattica (Figura 1) i soggetti che caratterizzano un contesto didattico: I'insegnante, I'allievo e il Sapere.

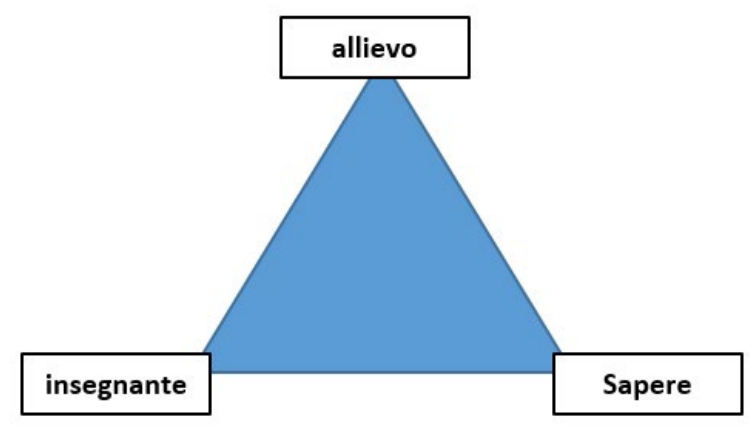

D'Amore e Fandiño Pinilla (2002) propongono una profonda analisi del triangolo e mostrano come la sua apparente semplicità nasconda una potenza teorica incredibile della quale più avanti, nel par. 4 del presente lavoro, cercheremo di dare dimostrazione.

Gli Autori, orchestrando e articolando contributi di diversi ricercatori, presentano una lettura analitica e specifica dei singoli elementi che compongono tale modello sistemico: i vertici che fungono da poli e i lati che evidenziano le relazioni tra coppie di poli. In particolare, D'Amore e Fandiño Pinilla caratterizzano ciascun polo, pensandolo come attrattore di studi specifici: la teoria degli ostacoli, le misconcezioni, aspetti epistemologici e ontologici della matematica legati all'apprendimento, le competenze, credenze e convinzioni ecc. A ciascun lato/relazione corrispondono attività ed elementi di riferimento: la devoluzione, la trasposizione didattica, l'implicazione personale (Brousseau, 1986, 2002; D'Amore, 1999; D'Amore \& Fandiño Pinilla, 2002). Nell'attuazione del processo didattico, dal Sapere, inteso come sapere accademico, si giunge al sapere competente dell'allievo. Tale percorso si scompone in una catena di trasformazioni, nitidamente descritta in Fandiño Pinilla (2002) che schematizziamo nella Figura 2: 
Figura 2

Catena di trasformazioni

che dal Sapere portano al

sapere competente (Fan-

diño Pinilla, 2002, p. 29).

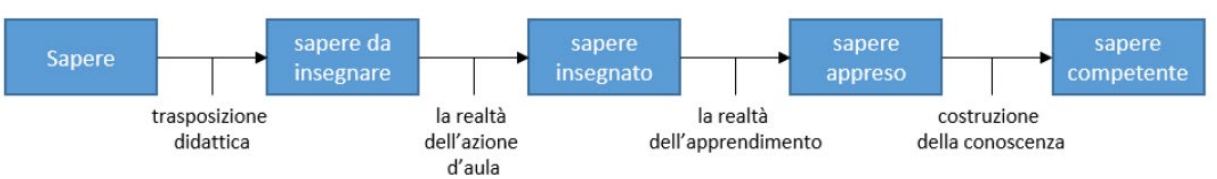

La prima trasformazione, la trasposizione didattica, è il processo gestito dalla professionalità dell'insegnante attraverso il quale si trasforma il sapere accademico in sapere da insegnare, accessibile all'alunno per la costruzione personale delle competenze matematiche (Fandiño Pinilla, 2002, 2003). Attraverso la realtà dell'azione d'aula, il sapere da insegnare si trasforma in sapere insegnato. II sapere insegnato si rivolge ad un allievo e la realtà dell'apprendimento di quell'allievo (con le sue convinzioni, concezioni, conoscenze pregresse ecc.) trasforma il sapere insegnato in sapere appreso. Il passaggio da sapere appreso a sapere competente mette in campo una serie di ulteriori elementi relativi allo sviluppo di competenza matematica che esulano dagli scopi del nostro studio e per i quali rimandiamo a Fandiño Pinilla (2003).

L'introduzione delle tecnologie digitali nei processi di I/A della matematica aumenta inevitabilmente la complessità del sistema didattico. Albano (2017) ed Albano et al. (2013), nello specifico caso dell'e-learning in matematica propongono un Tetraedro in cui i 4 vertici rappresentano i diversi elementi in gioco - la Matematica (M), lo Studente (S), il Tutor (T) e l'Autore (A) - e le 4 facce - MST, STA, MSA ed MTA - permettono di modellizzare ed analizzare le relazioni tra tali elementi. Per non appesantire il quadro teorico non faremo riferimento al Tetraedro. Ci preme, tuttavia una aspetto importante che emerge dalle riflessioni di Albano (2017) che mette in evidenza come la presenza di tecnologie digitali determini un'esplicita centralità dello studente nel processo di apprendimento, abilitando una dinamicità nei ruoli che lo studente stesso può assumere:

«Nella nostra visione sistemica, questo significa che i vertici del tetraedro non sono figure statiche, ma le consideriamo solo come posizioni, vale a dire come un elemento del sistema che può essere interpretato anche dallo Studente in alcune situazioni lungo il percorso di apprendimento. [...] A differenza dall'insegnamento classico, I'ingegneria didattica dovrebbe prendere in considerazione la possibilità per gli studenti di muoversi in altri/e vertici/posizioni del tetraedro. Questo aggiunge una dimensione di dinamicità nella quale I'apprendimento non emerge dalla fruizione di un prodotto preconfezionato, ma è l'esito di una costruzione nella quale gli studenti hanno la possibilità di compiere funzioni didattiche - supportate, guidate e supervisionate opportunamente - che influenzano la stessa organizzazione didattica».

(Albano, 2017, pp. 353-354, traduzione degli autori)

Nel presente studio faremo riferimento al Triangolo di Chevallard (nel seguito: Triangolo) la cui struttura triadica generale ci permetterà di rappresentare le diverse interazioni e relazioni che intercorrono tra le persone protagoniste dei due esempi che esamineremo nel par. 4. In tali esempi terremo conto della citata dinamicità che permette, soprattutto all'allievo di assumere posizioni diverse all'interno del modello. Infatti, il Triangolo si configura come una relazione triadica le cui variabili sono i vertici del triangolo, che di volta in volta si istanziano con l'assunzione di ruolo da parte di individui specifici e che in alcuni casi possono anche scindersi in istanziazioni diverse del ruolo generale da parte di più individui. 


\subsection{La classe intesa come società e l'interazione tra spazi di apprendimento fisici e spazi di apprendimento virtuali}

Seguiremo la proposta di interpretazione sociologica delle dinamiche d'aula di D'Amore (2005) e di Bagni e D'Amore (2005) e, come primo passo, focalizzeremo il significato del termine chiave società per il quale gli Autori si rifanno alla definizione sociologica. Si parla di società quando gli individui occupano un territorio comune, interagiscono tra loro, sanno di appartenere allo stesso gruppo, hanno - o si suppone abbiano - almeno una parte di cultura in comune. In ogni società, tra gli individui che ne fanno parte, si generano due tipologie di pratiche: quelle che si sostanziano dagli scopi costitutivi di quella società e quelle che traggono origine dall'adattamento all'appartenenza alla società stessa.

Secondo gli Autori, è possibile pensare alla classe - intesa come gruppo di persone che occupano uno stesso spazio di apprendimento fisico quale, ad esempio, l'aula scolastica o la scuola - come una specifica società di individui «la cui unità sociale è dovuta alla necessità sancita per legge della effettuazione di "pratiche" definite e in grande misura condivise» (D'Amore, 2005, p. 5). In tale società, che assume significato nel contesto scolastico e quindi costituisce un'istituzione sociale, alcuni individui assumono il ruolo di studenti ed altri il ruolo di insegnanti i quali, in tale ambito, rappresentano l'istituzione stessa.

Inquadrare una classe come società (nel seguito: società-classe) permette di raffinare la visione delle pratiche che in essa si realizzano. Infatti grazie alla lente sociologica proposta da D'Amore (2005) si può distinguere tra le pratiche funzionali che sono quelle che definiscono a priori la società-classe, e le meta-pratiche extrafunzionali, che sono più contestuali e legate al voler appartenere alla società stessa. L'Autore mette in evidenza che una società-classe, ha come scopo funzionale I'I/A però, non è detto che tutti gli individui che fanno parte di tale società-classe condividano gli stessi obiettivi ed abbiano le stesse prospettive. Si possono individuare le due tipologie di gruppi seguenti: quello degli individui con scopi, prospettive e pratiche funzionali alla specifica società di appartenenza e quello degli individui che privilegiano le meta-pratiche extrafunzionali come ad esempio quelle generate dalle dinamiche di contratto didattico definito da Brousseau (1986) come:

"costituito [...] dalle "abitudini specifiche del maestro attese dall'allievo" e dai "comportamenti dell'allievo attesi dal docente" e proprio la scelta del comportamento da tenere, da parte dell'alunno, diventa problematica poiché porta il soggetto ad interrogarsi su "cosa l'insegnante si aspetti che faccia"».

(Brousseau, 1986, p. 66)

Considerare un ambiente di I/A in cui I'uso di tecnologie digitali abilita l'interazione tra spazi di apprendimento fisici e spazi di apprendimento virtuali, rende necessarie ulteriori riflessioni. Infatti, tali condizioni modificano il contesto impattando sulle connotazioni degli elementi strutturali attorno ai quali ruota la definizione stessa di società e le idee di classe, di aula, di pratica e di meta-pratica. Ė ancora possibile individuare un territorio comune occupato dagli individui che popolano questo nuovo spazio di apprendimento ma si tratta di uno spazio multicomponenziale e ibrido per via della contemporanea presenza di una componente fisica e di una componente virtuale. Inoltre, sono ancora presenti delle interazioni degli individui tra loro e degli individui con l'ambiente - così come le modalità di espletazione delle pratiche nelle quali tali individui sono implicati - ma esse avvengono sia su un piano fisico, stretta- 
mente dipendente da vincoli percettivi e spazio-temporali, che su un piano virtuale nel quale l'individuo può controllare e gestire non solo gli aspetti spazio-temporali ma anche comunicazioni del tipo molti ad uno (many to one). Infatti, gli ambienti virtuali sono caratterizzati da una sorta di evanescenza dei confini spazio-temporali nei quali gli individui coinvolti prendono decisioni ed agiscono attraverso gli account. Da un punto di vista infrastrutturale, gli ambienti virtuali costringono (permettono) gli (agli) individui coinvolti a (di) rendere esplicito ciò che negli ambienti fisici potrebbe rimanere su un piano di inconsapevolezza o essere difficilmente praticabile.

Ad esempio, in un ambiente di I/A in cui spazi fisici e spazi virtuali interagiscono, lo spazio di apprendimento virtuale:

- può essere sfruttato per mettere in connessione individui appartenenti a spazi fisici disgiunti (per esempio classi fisiche diverse, scuole diverse), dando vita a nuove società, a loro volta specifiche e distinte dalle società di origine degli individui coinvolti, con le loro pratiche e meta-pratiche;

- può permettere ad esempio di: scindere esplicitamente l'individuo insegnante dalle azioni che la figura dell'insegnante compie in un contesto di l/A (progettare attività e situazioni, gestirle, valutare ecc.); isolare ciascuna di tali azioni, anche eventualmente predisponendo ambienti e/o account specifici per ciascuna; decentralizzare l'esecuzione di tali azioni distribuendole tra individui diversi; ecc. In questo senso, sono di grande interesse i vertici Autore e Tutor proposti nel modello del Tetraedro i quali evocano una particolarizzazione di possibili azioni espletate dagli attori in gioco in un contesto didattico. Tali scissioni e ridistribuzioni di compiti e responsabilità, costringono a ripensare, anche in vista di eventuali modifiche, non solo ai ruoli degli individui (insegnanti e studenti) che popolano la società-classe ma anche alle loro relazioni. Le modifiche, a loro volta, condizioneranno di conseguenza sia le forme di appartenenza dell'individuo alla società (pratiche) che i tentativi di adattamento (meta-pratiche), con un possibile impatto anche su ciò di pre-esistente nelle coinvolte società-classi localizzate nelle aule fisiche.

In definitiva, uno spazio di l/A virtuale si sostanzia nella dinamicità e flessibilità del Triangolo che permette di costituire società-classi seguendo criteri di raggruppamento contestuali e modificabili (Del Zozzo, 2019). Tali caratteristiche, inevitabilmente, impattano sull'idea di classe, quella di aula così come quella di pratiche e meta-pratiche, problematizzandole.

\subsection{Domande di ricerca}

II presente lavoro intende caratterizzare la contaminazione tra classe fisica e classe virtuale. In particolare, vogliamo analizzare le situazioni in cui uno spazio di I/A fisico viene contaminato da tecnologie digitali per la comunicazione con le quali si abilita un nuovo spazio di I/A. Sottolineiamo che l'implementazione di tecnologie digitali per la comunicazione in uno spazio di I/A fisico preesistente non può realizzarsi nell'immediato con un profondo ed efficace intreccio tra classe fisica e classe virtuale, ma piuttosto va guardato come una contaminazione della classe fisica da parte della classe virtuale e viceversa.

Per comprendere la natura e gli effetti di tale contaminazione sull'//A della matematica occorre rispondere alle seguenti domande di ricerca: 
D1: Come caratterizzare il sistema che emerge dalla contaminazione della classe fisica da parte della classe virtuale?

D2: Esistono dei tratti distintivi da monitorare per tracciare l'evoluzione dei processi di I/A nell'interazione tra la classe fisica e la classe virtuale? E se esistono, quali potrebbero essere?

Al termine del par. 4.3, dopo aver descritto ed analizzato i due esempi di implementazione, risponderemo a tali domande.

\section{Due esempi di implementazione}

Di seguito verranno descritti due esempi di dinamiche di I/A realizzati in spazi di apprendimento fisici che interagiscono con spazi di apprendimento virtuali. Entrambi gli episodi, sono esempi di comunicazione attraverso la tecnologia e non sono tratti da sperimentazioni specifiche con una propria progettazione e metodologia ma provengono da situazioni reali. II primo episodio coinvolge una classe seconda di Liceo Scientifico delle Scienze Applicate e il secondo episodio due studenti di una classe quinta di Liceo Classico. Ciascun esempio - che contestualizzeremo e del quale forniremo le specifiche metodologiche e strumentali di interazione tra il piano fisico e quello virtuale, inquadrando gli individui coinvolti e specificandone ruoli, azioni e comportamenti - verrà quindi interpretato alla luce del quadro teorico proposto. In entrambi i casi, la nostra analisi è a posteriori e si propone di mettere in relazione l'ambiente fisico con quello virtuale. Si tratta di dati che consideriamo particolarmente interessanti perché il ruolo dei ricercatori si è limitato solo alla loro raccolta e all'analisi senza entrare nel merito della progettazione delle attività e la loro realizzazione nel nuovo spazio fisico-digitale. Sono dati "puliti" che mettono in luce alcuni comportamenti spontanei di insegnanti e studenti in un contesto di I/A ibridato dalla presenza di tecnologie digitali per la comunicazione. Riteniamo pertanto che i due episodi siano adatti da un lato a mostrare esempi provenienti dalle pratiche d'aula quotidiane dall'altro a mostrare le potenzialità interpretative del nostro quadro teorico. Non avendo seguito una metodologia sperimentale standard, i dati a nostra disposizione sono per certi aspetti parziali ed è su quelli che baseremo le nostre analisi.

\subsection{Classe virtuale in Google Classroom \\ 4.1.1 Descrizione}

Scuola e classe: Seconda Liceo Scientifico Scienze Applicate.

Descrizione del contesto: Nell'anno scolastico 2017/2018, in cui è ambientato l'episodio descritto, la scuola ha ospitato una tirocinante laureata in matematica e specializzata nell'uso delle tecnologie per la didattica che ha effettuato un percorso di affiancamento di insegnanti e studenti nell'analisi, progettazione e implementazione delle tecnologie digitali per I'I/A. La scuola è registrata al servizio di Google della $G$ Suite for Education ${ }^{3}$ (GSE) e la tirocinante agisce come Amministratore. Da po-

3. https://support.google.com/a/answer/2856827?hl=it. 
Prospettive teoriche per lo studio della contaminazione tra ambienti di insegnamento/apprendimento fisici e virtuali / Agnese Del Zozzo e George Santi

che settimane $e^{4}$, alcuni docenti hanno avviato I'uso di Google Classrom ${ }^{5}$ (GC) come piattaforma di gestione delle classi virtuali. In questo esempio è coinvolta un'unica classe che ha iniziato ad utilizzare GC con la docente di matematica per la gestione logistica di un percorso didattico sui radicali realizzato nelle settimane precedenti. Al termine di tale percorso, la docente, su input della tirocinante, propone agli studenti di utilizzare lo Stream ${ }^{6}$ di GC come spazio per condividere dubbi, domande e difficoltà. La docente coinvolta insegna in questa classe già dal primo anno e l'unica tecnologia digitale da lei usata fino al momento dell'introduzione di GC era il registro elettronico per l'invio di compiti e la registrazione dei voti. Pertanto, I'introduzione della classe virtuale con GC si inserisce in un contesto didattico pre-esistente le cui specifiche (relazioni, dinamiche di contratto didattico, abitudini ecc.) sono già delineate in base al solo contesto della classe fisica.

Tecnologia digitale attraverso la quale si comunica: La componente software è GC con tutte le sue funzionalità. La componente hardware può essere il PC, il tablet o lo smartphone a seconda del dispositivo usato da chi interagisce nella piattaforma.

Persone fisiche ed account coinvolti: 26 persone fisiche di cui: 22 studenti della classe, 1 insegnante curricolare di matematica, 1 tirocinante, 2 docenti curricolari ma di altre discipline (passivi). 27 account, uno per ciascuna persona fisica più un account passivo, gestito dalla tirocinante, aggiunto in GC come studente fittizio con lo scopo di monitorare il corretto fluire delle attività in piattaforma anche dal lato studente. Al netto delle figure passive, considereremo solo 24 persone fisiche con i rispettivi 24 account. Nel seguito dell'esempio, l'etichetta $\mathrm{S}_{\mathrm{i}}$ simboleggerà un individuo-studente della classe.

Caratterizzazione dell'interazione tra il piano fisico e quello virtuale: La corrispondenza tra individui appartenenti alla classe fisica e la classe nell'ambiente virtuale è quasi uno ad uno, con l'unica eccezione della figura della tirocinante che appartiene in modo stabile solo al secondo contesto e che, nella classe fisica è stata presente in alcune occasioni sporadiche. La tirocinante non ha potere valutativo né un ruolo legato alla valutazione. Gli account in GC sono configurati con nome e cognome completi di tutte le persone coinvolte quindi esiste anche un'esplicita corrispondenza identitaria tra gli individui dell'ambiente fisico e gli account nell'ambiente virtuale.

Tabella 1

Individui che popolano la classe fisica e la classe in $\mathrm{GC}$

\section{Classe nell'ambiente virtuale}

2 insegnanti attivi (curricolare e tirocinante)

22 studenti

4. Rispetto ai giorni in cui si verifica l'episodio in esame.

5. https://support.google.com/edu/classroom/?hl=it\#topic=6020277.

6. Si tratta di uno spazio di comunicazione visibile a tutti i componenti di quella classe in GC, siano essi insegnanti o studenti. Per approfondire: punto di vista studenti al link https://support.google.com/edu/ classroom/answer/6020274?hl=it\&ref_topic=9049835; punto di vista insegnanti al link https://support. google.com/edu/classroom/answer/6020270. 
Prospettive teoriche per lo studio della contaminazione tra ambienti di insegnamento/apprendimento fisici e virtuali /

Agnese Del Zozzo e George Santi

Quando: I'episodio descritto si verifica tra il 24 e il 28 novembre del 2017.

\section{Cosa è successo:}

I passaggi verranno numerati per semplificarne il riferimento nel par. 4.1.2.

1. Nella classe fisica, l'insegnante annuncia la modalità d'uso di GC.

2. II 24 novembre, I'insegnante assegna degli esercizi sul registro elettronico assegnando in contemporanea un compito in GC con la seguente consegna: "Postare la foto di tutti gli esercizi di compito in cui avete avuto una difficoltà, nel caso non abbiate avuto alcuna difficoltà postare almeno un esercizio».

Tra il 26 e il 27 novembre:

3. $\mathrm{S}_{1}$ accoglie l'invito dell'insegnante di utilizzare la GC per aiutarsi nello svolgimento dei compiti a casa e posta nello Stream la foto della pagina del libro di testo, indicando due esercizi fonte di difficoltà.

4. $\mathrm{S}_{2}$ posta nello Stream la foto della pagina del proprio quaderno, con gli esercizi che ha svolto, accompagnati dal seguente messaggio di testo: «Foto esercizi per il 28/11 (non me ne viene nessuno dei tre)».

5. Altri 3 studenti seguono il comportamento di $S_{2}$ e postano nello Stream la foto della pagina del proprio quaderno con gli esercizi svolti.

6. Un altro studente, $S_{6}$, segue il comportamento di $S_{1}$ e posta nello Stream la foto della pagina del libro di testo senza inviare i propri tentativi.

7. Interviene nello Stream $S_{7}$ e scrive un Post con il seguente messaggio di testo, accompagnato dalla foto in Figura 3: "A me sono venuti se volete potete guardarli ho scritto in matita i passaggi che ho saltato così potete capirli».

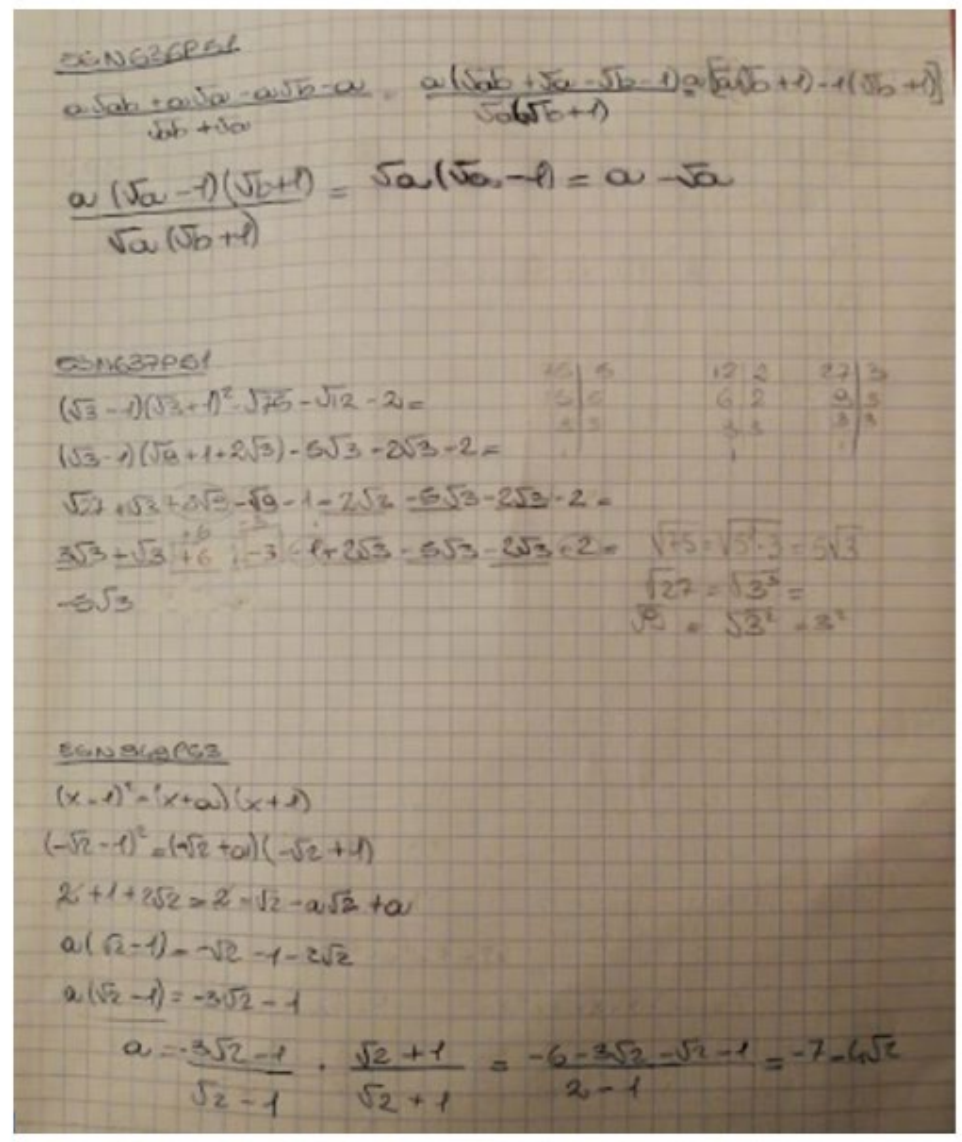


Prospettive teoriche per lo studio della contaminazione tra ambienti di insegnamento/apprendimento fisici e virtuali / Agnese Del Zozzo e George Santi

\section{8 novembre:}

8. L'insegnante risponde ringraziando con entusiasmo gli studenti che hanno condiviso il proprio quaderno, spiegandone l'utilità per individuare le difficoltà e per incoraggiare l'aiuto reciproco. $\mathrm{Ad} \mathrm{S}_{7}$, l'insegnante risponde ringraziando ed augurandosi che esercizi così dettagliati possano essere di aiuto a qualcuno. Nello Stream, non sono presenti risposte di studenti al Post di $\mathrm{S}_{7}{ }^{7}$

\subsubsection{Interpretazione}

Triangolo di Chevallard

L'interazione tra il piano fisico e il piano virtuale abilita la realizzazione di due configurazioni che possono essere messe in evidenza, o meglio portate in superficie, sfruttando il modello del Triangolo. In questo caso infatti, come schematizzato in Figura 4 possiamo distinguere due istanze di Triangolo: una per interpretare l'episodio nel contesto nella classe fisica $\left(T_{C F}\right)$ e una per interpretare l'episodio nel contesto nella classe virtuale in $\mathrm{GC}\left(\mathrm{T}_{\mathrm{GC}}\right)$.

Figura 4

Modellizzazione dell'esempio 1 sia nel contesto della classe fisica che in quello della classe in GC.

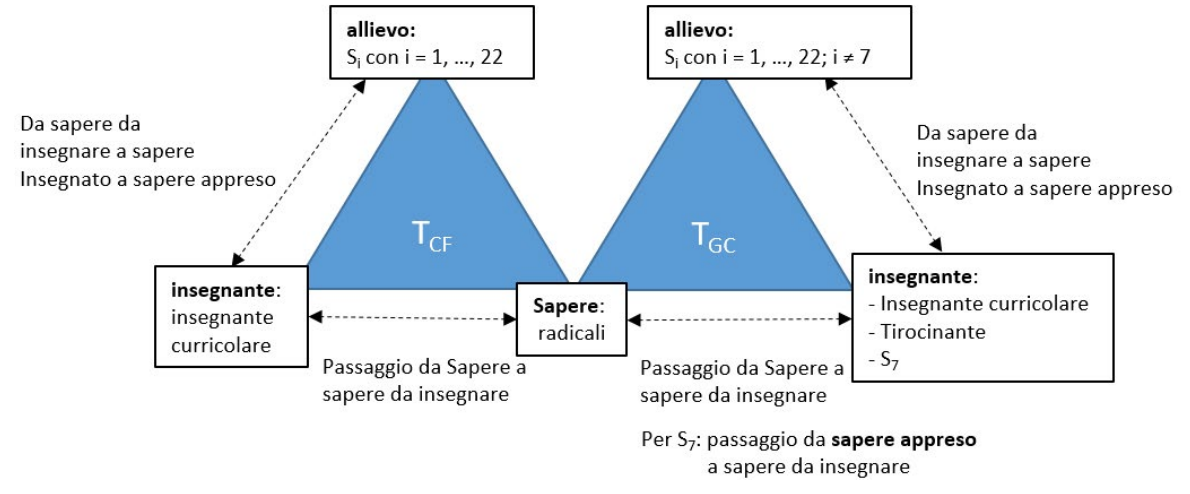

Se interpretiamo la situazione considerando solo l'insegnante curricolare, e quindi in riferimento al Triangolo di sinistra della Figura 4 possiamo riconoscere una sequenza didattica piuttosto standard. Se però consideriamo il Triangolo di destra la questione si problematizza in quanto la dinamicità della nuova situazione attivata dalla GC permette agli studenti di muoversi tra i vertici insegnante e allievo del Triangolo. S, raccoglie la proposta dell'insegnante fatta alla classe nello spazio fisico, la realizza nella GC assumendosi la responsabilità del proprio apprendimento e manifestando delle intenzioni didattiche nei confronti dei compagni. $\mathrm{S}_{7}$ in $\mathrm{T}_{G C}$ sta assumendo il ruolo di insegnante e opera una Trasposizione didattica dal proprio sapere appreso personale in sapere da insegnare adeguato ai suoi compagni (passaggio 7: «A me sono venuti se volete potete guardarli ho scritto in matita i passaggi che ho saltato così potete capirli»).

Istanze di Triangolo come $T_{G C}$ hanno caratteristiche peculiari sulle quali sarebbero necessari ulteriori studi. Infatti, non possiamo individuare un contratto didattico con clausole ed effetti in senso classico (D'Amore, 1999), in quanto, nell'insegnamento tra pari, il sistema di regole, comportamenti, aspettative, interpretazioni e convinzioni

7. Riteniamo utile mettere in evidenza che le funzionalità di GC, almeno fino al momento in cui è scritto il presente contributo, prevedono che quando ad inserire un Post nello Stream è uno studente non viene inviata una notifica, a differenza di quanto accade per i Post inseriti dall'insegnante (https://support.google.com/edu/classroom/answer/6141557? co=GENIE.Platform\%3DAndroid\&hl=it). Questo significa che è possibile che nessuno dei compagni si sia accorto in tempo utile del Post di $\mathrm{S}_{7}$. 
che caratterizza la nuova configurazione i-a-S ha una natura profondamente diversa da quella nella classe nello spazio fisico in cui è presente il docente di matematica. D'altra parte, I'apprendimento di $S_{7}$ come allievo nella $T_{C F}$ viene riattivato attraverso un processo di ri-costruzione personale del sapere in un'ottica comunicativa che sia di aiuto ai compagni che nella GC sono suoi allievi. Quindi $S_{7}$, con il suo agire inviando il Post di Figura 3, mette in campo processi tipici che riguardano la relazione tra insegnante e allievo sia in riferimento all'istanza $T_{G C}$, dove assume la posizione di insegnante (Trasposizione didattica) che in riferimento all'istanza $T_{C F}$, dove assume la posizione di allievo (devoluzione, implicazione personale, costruzione di conoscenza e rottura del contratto didattico).

\section{Interpretazione in chiave sociologica}

Da un punto di vista sociologico, sembrano emergere due società-classi distinguibili ma non separabili.

La prima società-classe è la società-classe fisica in cui: gli individui coinvolti sono i 22 studenti e la loro insegnante; il territorio comune occupato è fisicamente delimitato e le interazioni avvengono in tempi stabiliti a priori; le pratiche e le meta-pratiche si sono delineate nel tempo antecedente l'introduzione di GC. La società-classe fisica ha come pratica costitutiva I'I/A della matematica e in essa si sviluppano meta-pratiche alle quali partecipano sia gli allievi sia l'insegnante. Per esempio:

- Gli allievi cercano di soddisfare il bisogno di socializzazione, di consolidamento e riconoscimento della loro identità, di consenso.

- L'insegnante si preoccupa di soddisfare le richieste provenienti dalla noosfera, assolvere ai compiti burocratici, svolgere il programma, raccogliere un numero sufficiente di valutazioni; anche il docente agisce per scopi di tipo affettivo relazionale come per esempio, esercitare potere e controllo sulla classe, soddisfare le richieste della dirigenza, preoccuparsi della sicurezza e della sorveglianza, trasferire in aula, consapevolmente o inconsapevolmente, il proprio sistema di convinzioni sulla matematica, sugli studenti, sulla scuola e sulla società. Tutti questi aspetti interferiscono e/o possono confliggere con gli scopi costitutivi di questa società.

La seconda società-classe è la società-GC in cui: gli individui coinvolti sono i 22 studenti, la loro insegnante e la tirocinante; il territorio comune occupato è virtualmente delimitato dall'iscrizione alla GC creata dalla docente curricolare; le interazioni avvengono in modi e tempi personalizzati; la dinamicità del contesto e delle interazioni implica una dinamicità anche nelle pratiche e nelle meta-pratiche da cui deriva una configurazione pratiche e meta-pratiche flessibile e non stabile nel tempo. Inoltre, anche se le pratiche funzionali che definiscono la classe come società possono essere diverse, lo scopo costitutivo di questa seconda società è il medesimo della società-classe fisica: I'I/A della matematica. Per quanto riguarda le pratiche e le meta-pratiche della società-GC, ricordiamo che nel contesto considerato, tale società nasce successivamente alla società-classe fisica e nella società-classe fisica, prima dell'utilizzo di GC, le performance degli studenti venivano esaminate dall'insegnante solo in fase di verifica e valutazione. In generale, in questo episodio possiamo distinguere quattro tipi di comportamenti che, interpretati in termini di pratiche e meta-pratiche, evidenziano l'esistenza di diverse relazioni tra la società-classe fisica e la società-GC:

1. $S_{1}$ e $S_{6}$ postano la pagina del libro segnalando il testo degli esercizi per loro fonte di difficoltà; in questo modo localizzano la difficoltà senza esporsi mostrando sullo Stream il proprio tentativo di risoluzione. 
2. I 4 studenti da $S_{2}$ a $S_{5}$ postano nello Stream la foto del quaderno che contiene il proprio tentativo fallimentare; in questo modo localizzano la difficoltà e mostrano il processo di risoluzione implementato a tutti i componenti della società-GC.

3. $\mathrm{S}_{7}$ in risposta ai compagni, posta nello Stream la foto del quaderno che contiene i propri tentativi affermandone la correttezza ed esplicitando la propria intenzione didattica; così facendo non si limita ad esporre il proprio operato a tutti $i$ componenti della società-GC ma lo offre come modello per i propri pari.

4. Gli altri 15 studenti si astengono dal partecipare allo scambio nella GC, ciascuno con la propria motivazione (nessuna difficoltà, non voglia di esporsi, impossibilità strumentale di collegarsi ad internet ecc.).

Tali comportamenti, a nostro avviso, rivelano l'esistenza di forti relazioni tra pratiche e meta-pratiche nella società-classe fisica e quelle nella società-GC. L'agire di $S_{1}$ e $S_{6}$ può essere visto come una meta-pratica nella società-GC ereditata, per estensione, da una meta-pratica nella società-classe fisica generata dal timore della valutazione e da dinamiche di contratto didattico.

L'agire degli studenti del punto 2 da un lato è assimilabile ad una pratica costitutiva della società-GC dall'altro lato però può essere considerata una meta-pratica generata dal voler soddisfare la richiesta dell'insegnante di condividere i propri dubbi e le proprie difficoltà. Gli studenti, infatti, mostrano una parziale rottura del contratto didattico ma non mostrano implicazione personale e la volontà di costruire la propria conoscenza ponendo delle domande precise, attivando una discussione o condividendo la loro strategia risolutiva.

L'agire spontaneo di $\mathrm{S}_{7}$, che come già evidenziato manifesta implicazione personale e responsabilità, può essere considerato come una pratica caratterizzante la società-GC, coerente con il suo obiettivo di costruire e condividere conoscenza matematica. D'altra parte, il fatto che ciò che avviene nello Stream possa essere letto anche dall'insegnante, rende possibile interpretare questo stesso comportamento di $\mathrm{S}_{7}$ come una meta-pratica rispetto alla società-classe fisica. Infatti, $\mathrm{S}_{7}$ espone anche agli occhi dell'insegnante-valutatore il proprio operato dichiarandone la correttezza. Non è trascurabile nell'analisi delle pratiche e meta-pratiche della società-GC il fatto che 15 studenti non abbiano interagito. Non abbiamo elementi sufficienti per configurare tale comportamento come pratica o meta-pratica, ma è un gruppo di individui della società-GC qualitativamente e numericamente rilevante.

Dal dialogo tra la società-classe fisica e la società-GC emerge uno spazio di I/A ibrido: gli individui coinvolti sono i 22 studenti, la loro insegnante e la tirocinante; ciascuno è consapevole di occupare un territorio che è comune a tutti gli individui di tale società ma i confini di tale territorio subiscono delle modifiche continue date dalla contaminazione dei due ambienti; le interazioni e i flussi comunicativi da un lato sono maggiormente controllati e frutto di processi intenzionali espliciti ma, dall'altro lato, sono più spontanei perché ogni individuo ha la possibilità e lo spazio/tempo per esprimersi (o NON esprimersi) in modo personalizzato.

Inoltre, la discretizzazione tipica degli ambienti digitali rende possibile l'individuazione e la ricostruzione dell'agire del singolo, permettendo agli individui coinvolti di prendere decisioni più mirate e consapevoli. Infine, ciò che avviene in tale ambiente di I/A ibrido ha un'inevitabile relazione con le altre due società, entra in contatto con le pratiche e le meta-pratiche ivi esistenti rivelandole, mettendole in luce, in certi casi modificandole ma, in altri casi, cristallizzandole - si veda ad esempio l'episodio 2 descritto in Del Zozzo e Santi (2019).

In conclusione, l'introduzione della GC apre scenari comunicativi e risorse semiotiche 
molto diversi da quelli che caratterizzano la classe nell'aula fisica ma ci preme richiamare l'attenzione del lettore sul fatto che la qualità dell'interazione spontanea tra gli studenti in GC è, in questo esempio, scadente. Infatti, essa si riduce del tutto o quasi al caricamento di materiale su una piattaforma comune senza un vero scambio tra gli studenti. Alla luce della cornice teorica entro la quale stiamo svolgendo l'analisi, si tratta di un dato molto interessante. Occorre tenere innanzitutto presente che gli studenti hanno cominciato ad utilizzare la GC da poco tempo e non hanno maturato sufficiente esperienza per interagire efficacemente con questa tecnologia. In questa situazione, da un lato il comportamento degli studenti nella GC è indicativo di ciò che essi hanno mutuato dalla classe fisica tipicamente meta-pratiche extrafunzionali come ad esempio quelle riconducibili al contratto didattico. Dall'altro, la scelta dei materiali caricati non è neutra ma al contrario è molto eloquente. Infatti, per contrasto sono emersi i comportamenti interpretabili come pratiche funzionali e meta-pratiche extrafunzionali, che abbiamo descritto sopra, in cui ribadiamo l'interesse per il comportamento di $\mathrm{S}_{7}$ che dinamizza la relazione triadica del Triangolo passando dal ruolo di allievo nella società-classe fisica a quello di insegnante nella società-GC.

I dati a disposizione in questo esempio, anche a causa del breve intervallo temporale qui considerato, non ci danno informazioni sufficienti a delineare con precisione e profondità le dinamiche di I/A in questo spazio emergente. Tuttavia riteniamo che sia una direzione da indagare in quanto le pratiche e le meta-pratiche delle due società in gioco potrebbero non essere isomorfe ma, di certo entrano tra loro in relazione, contaminandosi.

\subsection{Chat privata tra studenti su Whatsapp}

In questo esempio viene descritto ed analizzato uno scambio di messaggi a tema matematico tra due compagni di classe, $\mathrm{S}_{1}$ ed $\mathrm{S}_{2}$, avvenuto nella loro chat privata di Whatsapp. L'accesso a tali dati è stato possibile perché uno degli autori del presente articolo conosce personalmente, in ambito privato, uno dei due studenti protagonisti dello scambio e gliene ha fatto esplicita richiesta. In risposta a tale richiesta, i due ragazzi si sono consultati e con grande generosità hanno accolto il nostro invito, autorizzandoci a sbobinare e trascrivere le porzioni della loro chat privata in cui discutevano di matematica. Lo scambio qui riportato ed analizzato è avvenuto circa un anno e mezzo prima rispetto a quando ai ragazzi è stato chiesto il permesso per potervi accedere ed è stato realizzabile perché uno dei due studenti non aveva svuotato la memoria delle proprie chat. Nel momento in cui lo scambio è avvenuto, i due ragazzi non sapevano né immaginavano né potevano ipotizzare che qualcuno di esterno avrebbe potuto leggere/vedere/ascoltare la loro conversazione. Si tratta di un'analisi a posteriori di uno scambio comunicativo privato autenticamente avvenuto tra $\mathrm{S}_{1}$ ed $\mathrm{S}_{2}$.

\subsubsection{Descrizione}

Scuola e classe: Ultimo anno del Liceo Classico.

Descrizione del contesto: La classe si sta preparando per l'ultima prova di verifica di matematica prima dell'esame di Stato e l'insegnante ha assegnato alcuni esercizi di ripasso. Alcuni studenti della classe affiancano il lavoro a scuola con delle lezioni pomeridiane in gruppo tenute da una stessa persona - diversa dalla loro docente 
Prospettive teoriche per lo studio della contaminazione tra ambienti di insegnamento/apprendimento fisici e virtuali / Agnese Del Zozzo e George Santi

curricolare. L'episodio considerato è tratto da una chat privata su Whatsapp tra due studenti, $\mathrm{S}_{1}$ ed $\mathrm{S}_{2}$, che condividono sia l'insegnante curricolare (sono compagni di classe) che l'insegnante privato.

Tecnologia digitale attraverso la quale si comunica: La componente software è Whatsapp con tutte le sue funzionalità. La componente hardware è lo Smartphone.

Persone fisiche ed account coinvolti: 4 persone fisiche: i due studenti $\mathrm{S}_{1}$ ed $\mathrm{S}_{2}$ l'insegnante privato (presente implicitamente) e un'ulteriore figura adulta familiare che per $S_{2}$ è un riferimento competente in matematica (presente implicitamente), 2 account su Whatsapp di $\mathrm{S}_{1}$ ed $\mathrm{S}_{2}$.

Caratterizzazione dell'interazione tra il piano fisico e quello virtuale: $\mathrm{S}_{1}$ ed $\mathrm{S}_{2}$ si accordano per fare tra loro una chiamata di esercitazione su Skype. Lo stesso giorno, $S_{1}$ partecipa ad una lezione con l'insegnante privato, a cui $\mathrm{S}_{2}$ è assente.

$\mathrm{S}_{1}$ ed $\mathrm{S}_{2}$ si sentono tramite Whatsapp il giorno seguente per aggiornarsi sulla lezione effettuata da $S_{1}$ con l'insegnante privato.

Tabella 2

Individui che popolano i tre ambienti fisici (la

classe fisica istituzionale,

quella privata e il con-
testo familiare) e che

comunicano tramite la

chat di Whatsapp.

\begin{tabular}{|l|l|l|l|}
\hline $\begin{array}{l}\text { Classe fisica } \\
\text { istituzionale }\end{array}$ & $\begin{array}{l}\text { Classe fisica } \\
\text { privata }\end{array}$ & $\begin{array}{l}\text { Contesto familiare } \\
\text { privato }\end{array}$ & $\begin{array}{l}\text { Interazione su } \\
\text { Whatsapp }\end{array}$ \\
\hline $\begin{array}{l}1 \text { insegnante } \\
\text { curricolare }\end{array}$ & $\begin{array}{l}1 \text { insegnante privato } \\
4 \text { studenti della stessa } \\
\text { classe (non sempre } \\
\text { tutti presenti) }\end{array}$ & $\begin{array}{l}1 \text { familiare adulto } \\
\text { riferimento competen- } \\
\text { te in matematica }\end{array}$ & 2 studenti \\
1 studente & \\
\hline
\end{tabular}

Quando: I'episodio descritto si verifica tra il 23 e il 24 maggio del 2018.

\section{Cosa è successo:}

I passaggi verranno numerati per semplificarne il riferimento nel par. 4.2.2.

1. La conversazione viene avviata da $S_{1}$ che informa $S_{2}$ che durante la lezione con I'insegnante privato ha svolto tutti gli esercizi; $S_{2}$, che stava in quel momento svolgendo gli stessi esercizi con la figura adulta familiare che per $\mathrm{S}_{2}$ è un riferimento competente, chiede ad $\mathrm{S}_{1}$ : «Mi manderesti le spiegazioni??».

2. $\mathrm{S}_{1}$ invia ad $\mathrm{S}_{2}$ cinque foto di pagine del proprio quaderno con il lavoro svolto durante la lezione privata del giorno precedente. Tra gli esercizi svolti inviati, c'è anche il seguente (il n. 4 di p. 1484) quesito a risposta multipla:
La funzione $f(x)=-x^{5}+2 x^{3}$
A. Ha solo un flesso orizzontale
B. Ha un flesso orizzontale e due obliqui
C. Ha tre flessi orizzontali
D. Ha tre flessi obliqui
E. Non ha flessi

La risoluzione di tale esercizio da parte di $\mathrm{S}_{1}$ è riportata in Figura 5: 


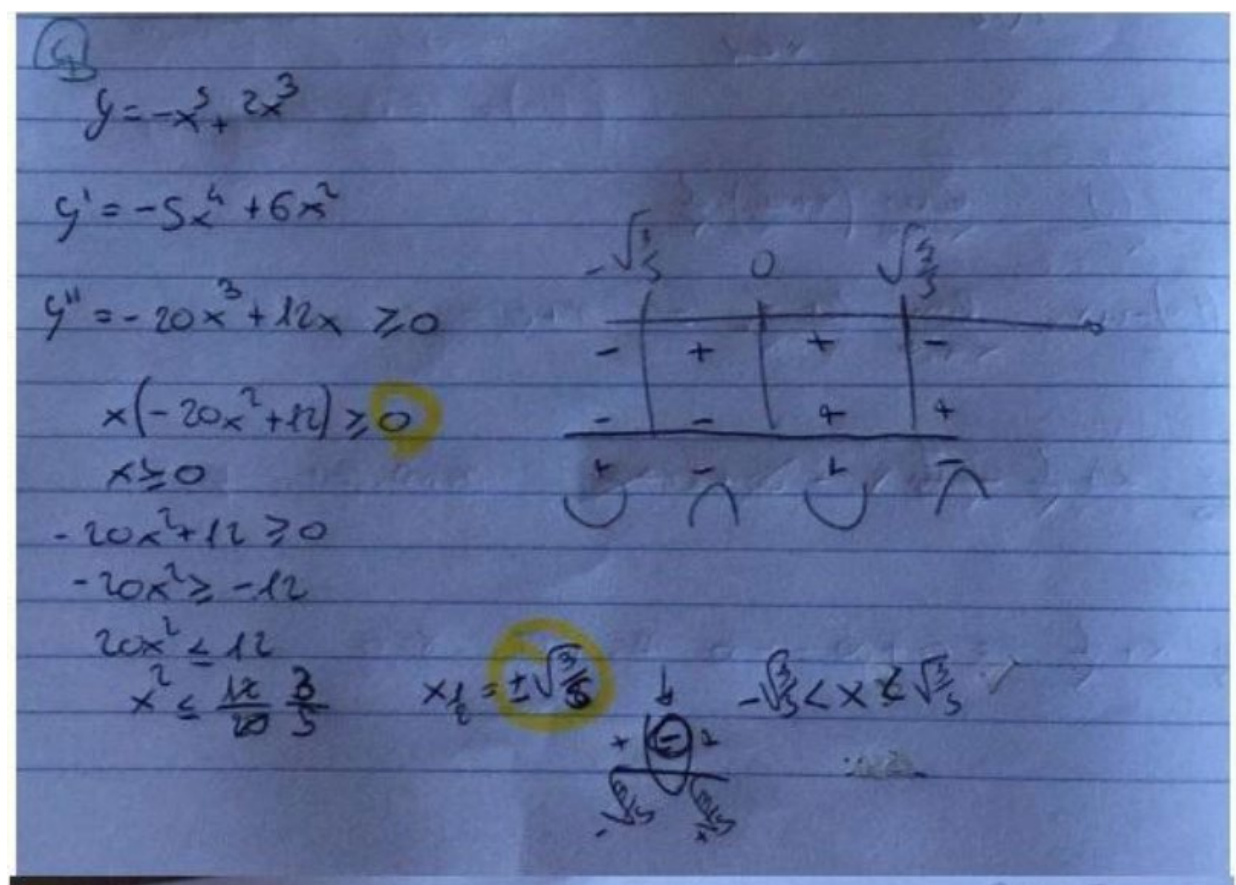

Figura 5

Estratto delle foto inviate da $S_{1}$ che contiene la soluzione del quesito a risposta multipla sui punti di flesso di una funzione data.

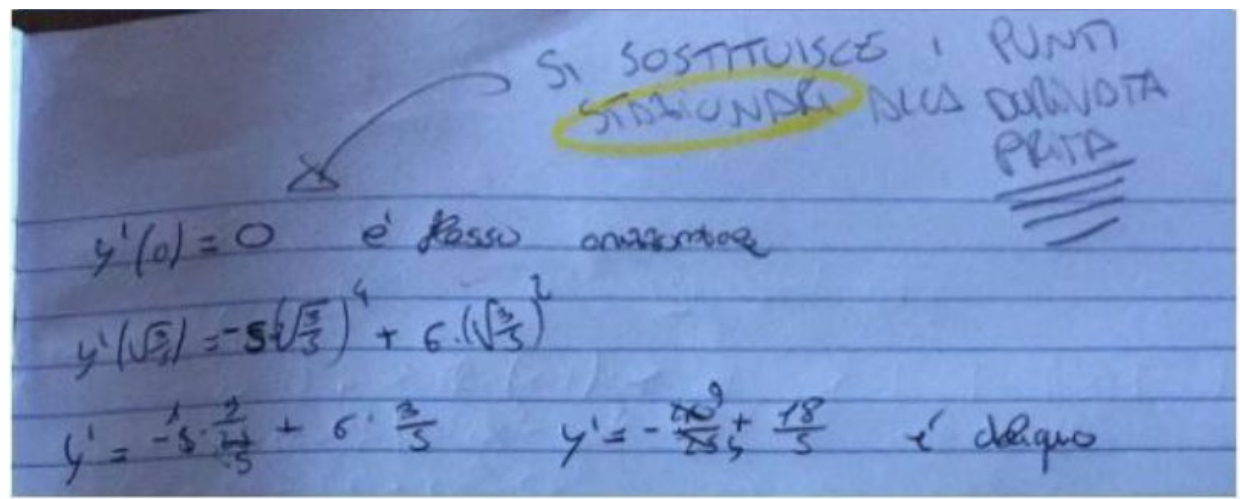

3. Dopo altri scambi di messaggi che non riguardano questioni matematiche, $S_{2}$ manda il seguente messaggio audio:

«L'esercizio di pagina 1484, il numero 4, quando devi svolgere la funzione e trovare i massimi i minimi i flessi e va bè, a un certo punto tu hai fatto la tabella quella dei segni e ci hai messo solo... cioè io... come faccio a capire... mmm... cioè quali sono i mi... cioè nel senso... dimmi che hai capito».

Tale messaggio è accompagnato con l'immagine del quaderno di $\mathrm{S}_{1}$ in cui $\mathrm{S}_{2}$ ha cerchiato una parte (Figura 6): 
Figura 6

Immagine che $\mathrm{S}_{2}$ invia per accompagnare l'audio trascritto in 3.

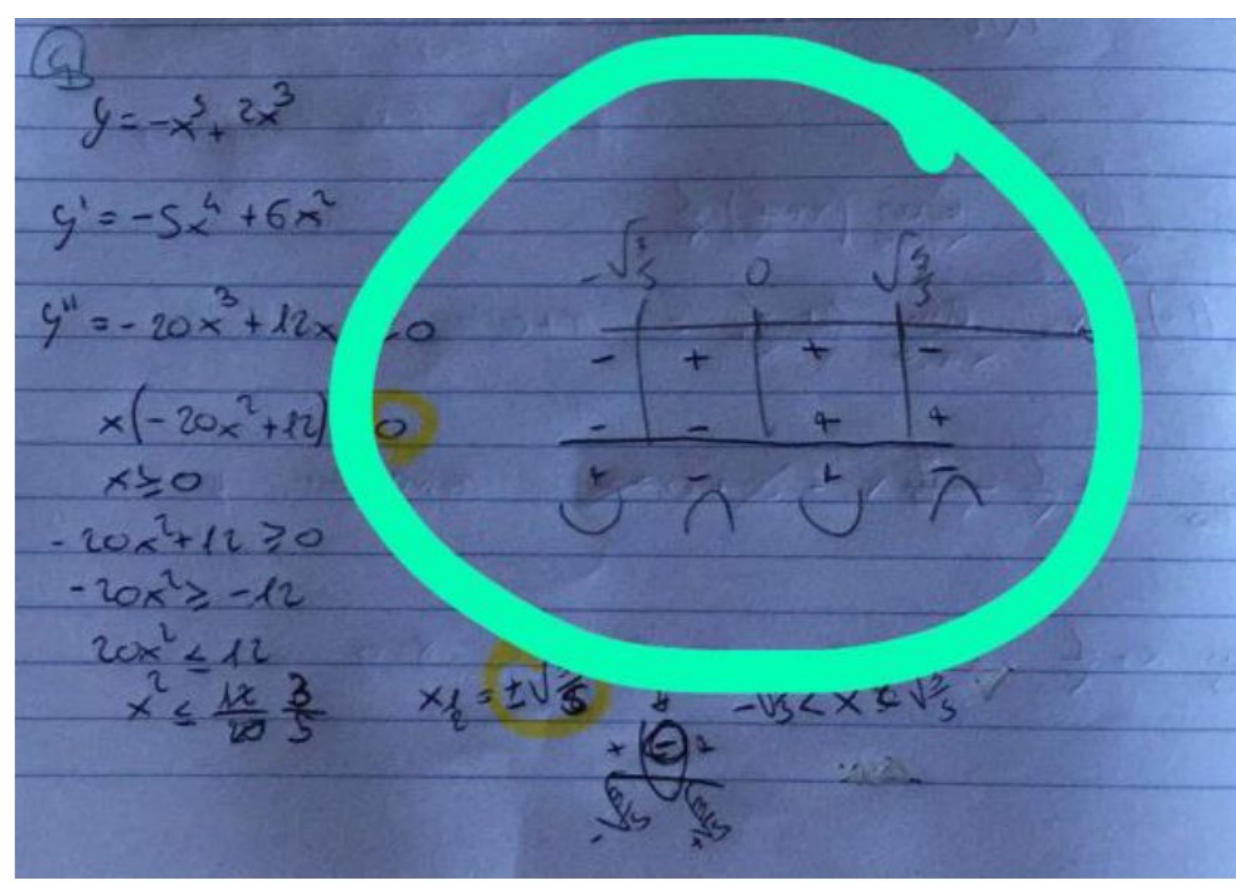

4. $\mathrm{S}_{1}$ risponde con il messaggio audio seguente:

«mmm no, non ho capito però ok te lo provo a spiegare, in pratica i massimi e minimi li trovi con la derivata prima. Le concavità con la derivata seconda. siccome il 4 ti cercava i flessi cioè i flessi le concavità li trovi con la derivata seconda quindi devi fare prima la derivata prima poi fai la derivata seconda e sulla derivata seconda fai lo studio del segno. Poi quando è maggiore la concavità è verso l'alto, quando è minore la concavità è verso il basso quindi siccome cambia concavità c'è un flesso, ok? Oooh. Poi una volta fatto questo, per capire se è un flesso orizzontale o se flesso obliquo devi fare quello che t'ho scritto con la matita nella foto cioè vado a sostituire i punti stazionari, che sarebbero 0 più o meno radice di tre quinti, alla derivata prima [enfasi con tono di voce], se riporta 0 è flesso orizzontale, capito? Infatti andando a sostituire 0 nella derivata è uguale $\mathrm{a} 0$ ed è flesso orizzontale. Se vado a sostituire più o meno radice di tre quinti mi ripor... non riporta 0 e quindi è... obliquo. Sia per più sia per meno. Quindi [enfasi con tono di voce] è un flesso orizzontale e due obliqui».

5. $\mathrm{S}_{2}$ scrive «Ok perfetto» e poi, dopo 5 minuti chiede «La 6 invece?» ${ }^{8}$

«Siccome devi andare a trovare quale di quelle, le funzioni lì sotto.. eh.. rispetta quei punti vai per esempio a.. tr... g...[borbottio incomprensibile, sembra iniziare diverse parole senza pronunciarne interamente nessuna] il primo punto è il dominio più o meno quattro cioè $x$ diverso da più o meno quattro [tono interrogativo] allora vai a vedere in queste cinque funzioni quale c'ha quella con

8. L'esercizio a cui si riferisce è un quesito a risposta multipla in cui bisogna decidere quale tra le cinque funzioni elencate è quella che soddisfa una serie di condizioni prestabilite. 
il dominio diverso da più o meno quattro. Quindi tipo la A non può essere, la $E$ non può essere, la $C$ neanche. Quindi già tu da queste ehm... intanto ne escludi 3 soltanto per il dominio. Quindi il dubbio è tra la B e la D. Ehm "Interseca l'asse $x$ nei punti $A$ e $B$ " [sembra leggere] allora vai a fare l'intersezione con l'asse y di $B$ e di $D$ per esempio e tutte e due puoi vedere, se lo fai, che tutte e due intersecano l'asse x nei punti A e B. Cioè tutti e due intersecano lì. Quindi devi andare a vedere con il punto 3 . "Ha come asintoto verticale le rette $x=4$ e $x=-4$ " [sembra leggere] fai il limite di $x$ che tende a 4 e... ehm... il limite di $x$ il limite di $x$ che tende $a$ il limite di $x$ che tende $a-4$ e anche sia la $B$ che la $D$ quindi vai a vedere il punto 4: "Ha come asintoto orizzontale la retta $y=2$ " quindi fai il limite di $x$ che tende a infinito di per esempio $B$ de... della funzione $B$ e vedi che è infinito fratto infinito e quindi... ehm... non è e l'asintoto orizzontale ce l'ha la D. E quindi è la D. Capito? Cioè vai per esclusione, inizi a fare i punti 1, 2, 3 e 4 alle funzioni e-e li escludi man mano che vai».

7. $\mathrm{S}_{2}$ scrive «Ok grazie» e la conversazione si conclude.

\subsubsection{Interpretazione}

\section{Triangolo di Chevallard}

In questo esempio, insegnante, allievo e Sapere entrano in relazione in 4 configurazioni differenti rappresentati nella Figura 7. Abbiamo una prima istanza del Triangolo che interpreta il contesto della classe fisica ( $T_{C F}$ in alto a sinistra nell'immagine); poi I'analisi richiede di considerare anche:

- una seconda istanza del Triangolo che rappresenta la relazione triadica tra l'insegnante privato, il Sapere (lo studio di funzioni) e l'allievo $\mathrm{S}_{1}\left(\mathrm{~T}_{\mathbb{P}}\right.$ in alto a destra nell'immagine);

- una terza istanza del Triangolo individuato dai vertici Sapere (lo studio di funzioni), I'insegnante che in questa situazione è rappresentato dal familiare adulto riferimento competente per $S_{2}$ e l'allievo $S_{2}\left(T_{R C}\right.$ in basso a destra nell'immagine);

- una quarta istanza del Triangolo individuato dai vertici Sapere (lo studio di funzioni), l'insegnante che in questa situazione è rappresentato da $S_{1}$ e l'allievo $S_{2}$ (TwH in basso a sinistra nell'immagine); questa situazione è simile a quella creata da $S_{7}$ nell'esempio precedente con una differenza cruciale: non è presente l'insegnante curricolare ma, di fatto, $S_{1}$ si comporta come se fosse l'insegnante, $S_{2}$ come un allievo e c'è un Sapere in gioco.

Sempre in riferimento alla Figura 7, ci sembra importante puntualizzare che il diverso punto di partenza del Sapere della figura di insegnante in ciascun triangolo dipende dalla persona che sta in quel contesto assumendo quel ruolo. Ad esempio, nel caso $\mathrm{di}_{\mathrm{RC}}$ il familiare adulto competente è una persona di qualche anno più grande che ha già terminato la scuola superiore. Pertanto, per quanto il suo sapere appreso personale possa essere vicino a quello accademico, non può essere considerato tale. 
Figura 7

Interpretazione dell'esempio 2 con diverse istanziazioni del Triangolo.

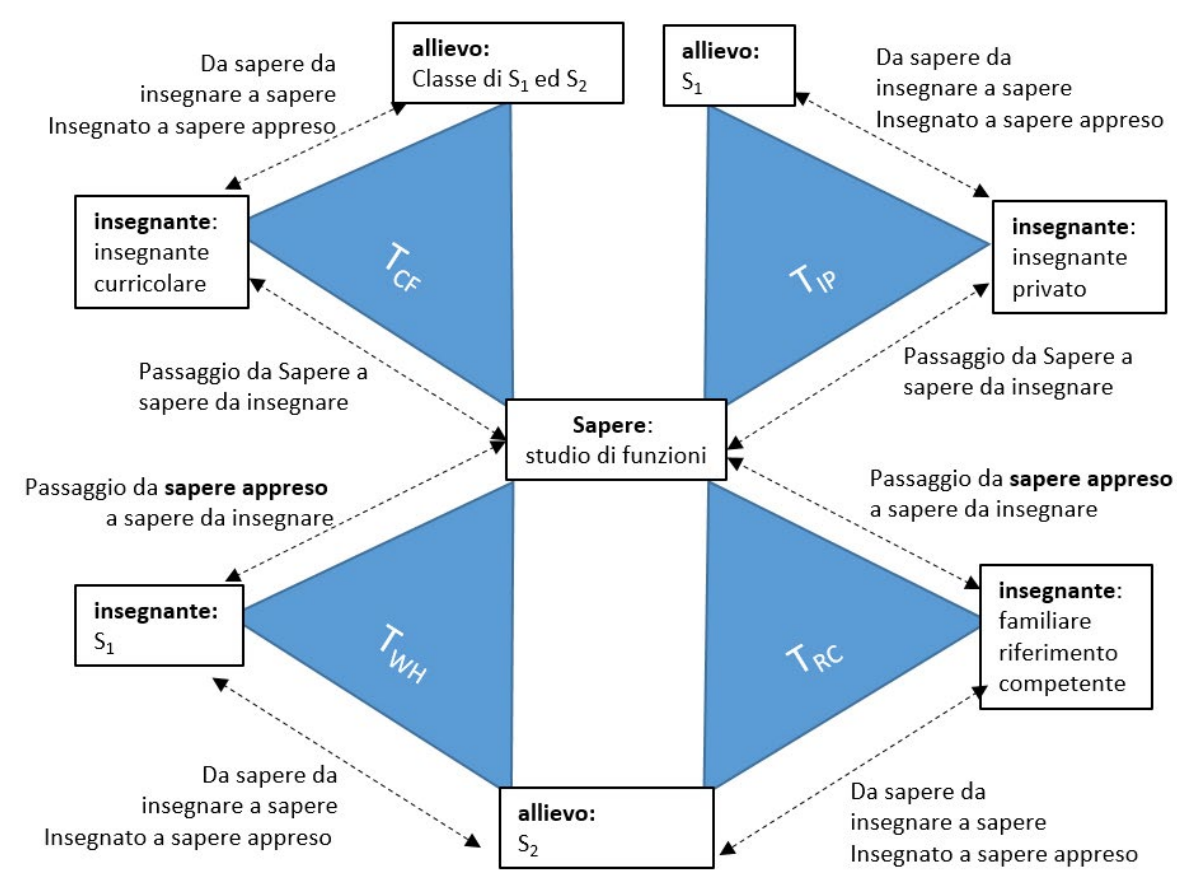

Nell'esempio che stiamo considerando, i triangoli descritti sopra non sono intrecciati come lo erano quelli dell'esempio del par. 4.1: nelle quattro istanze di triangoli il ruolo di insegnante è assunto da quattro figure differenti, di cui una è uno studente; inoltre, I'insegnante di matematica curricolare qui è presente solo nella classe fisica mentre nel caso precedente era presente anche nella GC; infine, solo alcuni studen$\mathrm{ti}, \mathrm{S}_{1}$ e $\mathrm{S}_{2}$, della classe fisica istituzionale sono presenti in tutte le triangolazioni di Chevallard. Analizzeremo solo il contesto di apprendimento che si configura dalle relazioni tra $S_{1}$ e $S_{2}$ nella chat di Whatsapp. L'insegnante curricolare non è presente ma, di fatto, quella che si crea è una situazione di alta rilevanza didattica. Nello scambio riportato, i due ragazzi discutono su due esercizi e possiamo organizzare il contenuto in due parti: dal passaggio 1 al passaggio 4 (compresi) la discussione riguarda il primo esercizio; dal passaggio 5 al passaggio 7 (compresi) $\mathrm{S}_{1}$ ed $\mathrm{S}_{2}$ parlano del secondo esercizio.

Nell'intero scambio, appare emblematica la figura di $S_{1}$ : comunica la sua disponibilità ad aiutare $\mathrm{S}_{2}$ nella risoluzione di alcuni esercizi ed attua la devoluzione degli obiettivi didattici che $\mathrm{S}_{2}$ accetta implicandosi personalmente nell'attività matematica. Come nel caso di $S_{7}$ nell'episodio precedente, assistiamo ad una ri-costruzione del sapere appreso attivata da $\mathrm{S}_{1}$ nell'intento di aiutare l'apprendimento di $\mathrm{S}_{2}$, riscontrando il medesimo atteggiamento nel modificare il proprio lavoro per massimizzare la portata comunicativa del materiale condiviso (passaggio 4: «[...] devi fare quello che t'ho scritto con la matita nella foto [...]»). D'altra parte, nel triangolo $T_{W H}$ dove $S_{1}$ assume il ruolo di insegnante, questo agire può essere interpretato come una sorta di Trasposizione didattica che non è quella classica che comincia con un Sapere sapiente e termina con il sapere competente dall'allievo, ma la sequenza inizia dal sapere appreso da $S_{1}$ nella combinazione dei triangoli $T_{C F}$ e $T_{\mathbb{P}}$. L'intenzione didattica di $S_{1}$ raggiunge $S_{2}$ che manifesta un atteggiamento critico e la volontà di imparare. $\mathrm{S}_{2}$, nella discussione che riguarda il primo esercizio, espone la sua difficoltà in un linguaggio - che è al di fuori dal gergo tipico degli atteggiamenti contrattuali, gergo 
che D'Amore (1999) chiama "matematichese" - apparentemente incomprensibile a chi non appartiene alla relazione comunicativa tra $S_{1}$ e $S_{2}$ ma accessibile a $S_{1}$. Vogliamo portare all'attenzione del lettore il ruolo attivo che $S_{2}$ assume nello scambio con $S_{1}$ prendendosi anche la responsabilità di intervenire sui materiali di $S_{1}$, operazione improbabile all'interno delle forme contrattuali tipiche dei sistemi didattici che coinvolgono la presenza dell'insegnante curriculare, che potrebbe attivare aspettative e effetti noti in letteratura.

Mettiamo in evidenza come l'utilizzo delle tecnologie digitali sia essenziale nel concretizzare questa configurazione didattica e queste forme di comunicazione - la possibilità di fotografare le pagine del quaderno, intervenire semioticamente sul testo originario disponibile in forma digitale, inviare nella rete tali documenti, potrebbero sembrare operazioni scontate, ma invitiamo il lettore a immaginare come si sarebbe sviluppata la dinamica di questo episodio senza le potenzialità offerte dalle tecnologie digitali. Inoltre, da un punto di vista della ricerca, la natura di queste tecnologie digitali ha permesso di accedere ad uno scambio spontaneo tra gli allievi, altrimenti accessibile solo al prezzo di attivare le ineliminabili interferenze del contratto sperimentale (D'Amore, 1999).

Nel discutere in merito al secondo esercizio, invece, la situazione non è connotabile con gli stessi elementi di implicazione personale e volontà di costruire conoscenza da parte di $\mathrm{S}_{2} . \mathrm{S}_{2}$ infatti si limita ad indicare un esercizio senza manifestare desiderio e volontà di capire una specifica porzione di Sapere - ricordando il comportamento di $S_{1}$ ed $S_{6}$ nel primo esempio descritto nel par. 4.1. Probabilmente, a differenza di quanto avvenuto nella prima parte della comunicazione, sono intervenuti obblighi didattici come la consegna degli esercizi, I'argomento dell'esercizio come possibile oggetto di un'interrogazione, I'ansia per l'avvicinarsi della verifica ecc. Assistiamo ad uno scivolamento da parte di $\mathrm{S}_{2}$ verso atteggiamenti che sembrano guidati da dinamiche di contratto didattico, quasi ad indicare l'inevitabilità del contratto didattico ereditato dalla classe fisica. Tuttavia, con i dati a nostra disposizione, non possiamo spingerci oltre in questa analisi.

\section{Interpretazione in chiave sociologica}

A nostro avviso, in questo episodio è possibile distinguere due società-classi e caratterizzare l'interazione tra $S_{1}$ ed $S_{2}$ in termini di pratiche e meta-pratiche. Da un lato abbiamo la società-classe fisica nell'istituzione scuola, popolata dall'insegnante curricolare e tutti gli studenti della classe. II territorio comune occupato è fisicamente delimitato e le interazioni avvengono in tempi stabiliti a priori. Dall'altro lato, abbiamo la società-classe privata non istituzionale, popolata dall'insegnante privato e da tutti gli studenti di quella classe che seguono le sue lezioni. Anche in questo caso, il territorio comune occupato è fisicamente delimitato e le interazioni avvengono in tempi delimitati ma concordati di volta in volta tra i membri della società. Le due società-classi, pur coinvolgendo individui diversi in territori diversi, condividono lo stesso scopo funzionale: I'I/A della matematica. In entrambe le società le pratiche e le meta-pratiche, si sono delineate nel tempo. In questo scenario, $\mathrm{S}_{1}$ ed $\mathrm{S}_{2}$ sono due individui che appartengono ad entrambe le società-classi ed esiste tra loro il rapporto di amicizia tra pari che, in quanto tale, è completamente centrato sugli interessi degli individui coinvolti.

Nell'esempio considerato, lo scambio comunicativo tra i due studenti sui due esercizi si realizza in uno spazio digitale intimo - che non è, di per sé, una società-classe ma 
risente delle pratiche e delle meta-pratiche delle due individuate sopra - dedicato alla loro relazione di amicizia. In tale ambito privato, lo scambio di battute sul primo esercizio presenta una differenza sostanziale rispetto a quanto avviene per il secondo. Infatti, il primo scambio nasce da un dubbio preciso attorno al quale $\mathrm{S}_{2}$ formula una domanda specifica e centrata su di sé (passaggio 3: «io come faccio a capire $[\ldots] »)$. II secondo scambio invece avviene sempre su input di $S_{2}$ ma la domanda presenta dei toni diversi rispetto alla precedente (passaggio 5: «La 6 invece?») e sembra quasi sottintendere un impersonale "come si fa".

Naturalmente da un punto di vista della relazione amicale tra $\mathrm{S}_{1}$ ed $\mathrm{S}_{2}$ tale differenza non esiste poiché entrambi gli scambi rientrano in un'interazione di aiuto perfettamente coerente con il rapporto di amicizia tra i due.

Tuttavia, interpretando questa interazione in termini di pratiche e meta-pratiche rispetto alla società-classe fisica cui entrambi appartengono, potremmo dire che mentre nel primo scambio, in cui $S_{2}$ si implica personalmente e manifesta l'intenzione di capire, si crea un'assonanza con le pratiche funzionali della società-classe, nel secondo emerge una sorta di dissonanza. Infatti, $\mathrm{S}_{2}$ perde il suo ruolo attivo e sembra rimanere sullo sfondo della propria domanda: ecco che, come già osservato, si realizza uno scivolamento verso una meta-pratica generata da dinamiche di contratto didattico.

Qui, come nell'esempio precedente, le due società-classe sono a intersezione non vuota.

\subsection{Risposta alle domande di ricerca}

D1: Come caratterizzare il sistema che emerge dalla contaminazione della classe fisica da parte della classe virtuale?

R1: Per caratterizzare il nuovo sistema, occorre individuare le possibili istanziazioni delle variabili in i-a-S che concorrono alla realizzazione della relazione triadica rappresentata dal triangolo. Per esempio, nell'episodio del par. 4.1 abbiamo individuato 2 possibili istanziazioni del Triangolo (Figura 4). La complessità delle relazioni che intercorrono tra i protagonisti dell'episodio del par. 4.2 ha richiesto di introdurre 4 schemi triadici.

Un altro elemento da prendere in considerazione è l'individuazione e la caratterizzazione delle società-classi in gioco. In particolare, per ciascuna società-classe occorre riconoscere gli individui/account, i territori e le "regole di interazione" tra individui/ account. Per esempio, nel primo episodio abbiamo individuato due società-classe:

- La classe fisica che è composta dagli studenti e dall'insegnante che agiscono in un territorio delimitato nello spazio e nel tempo secondo le regole tipiche del contesto d'aula.

- La GC che è composta da 24 individui/account, 22 studenti, l'insegnante e la tirocinante. II territorio non ha confini spaziali e temporali definiti. Essi dipendono dalle scelte degli individui e dalle caratteristiche della tecnologia utilizzata. Le regole di interazione sono determinate da accordi tra gli individui e i vincoli dettati dalla tecnologia, software e hardware.

Nel secondo episodio abbiamo individuato due società:

- Da un lato abbiamo la società-classe fisica nell' istituzione scuola, popolata dall'insegnante curricolare e tutti gli studenti della classe. II territorio comune occupato è fisicamente delimitato e le interazioni avvengono in tempi stabiliti a priori.

- Dall'altro lato, abbiamo la società-classe privata non istituzionale, popolata 
dall'insegnante privato e da tutti gli studenti di quella classe che seguono le sue lezioni. Anche in questo caso, il territorio comune occupato è fisicamente delimitato e le interazioni avvengono in tempi delimitati ma concordati di volta in volta tra i membri della società.

Osserviamo che le società-classi dei due episodi, nonostante coinvolgano individui diversi in territori diversi, condividono lo stesso scopo funzionale: I'I/A della matematica.

D2: Esistono dei tratti distintivi da monitorare per tracciare l'evoluzione dei processi di I/A nell'interazione tra la classe fisica e la classe virtuale? E se esistono, quali potrebbero essere?

R2: Per rispondere alla seconda domanda occorre individuare fattori di tipo dinamico per seguire l'evoluzione dei processi di I/A. Tali fattori sono legati alle lenti teoriche che stiamo utilizzando per interpretare le contaminazioni reciproche tra classe fisica e classe virtuale.

Per quanto concerne il Triangolo, occorre osservare la dinamicità delle istanziazioni della relazione triadica rispetto alle variabili i-a-S. Come abbiamo indicato nel par. 3, I'introduzione di classi virtuali comporta una dinamicità nelle possibili istanziazioni del Triangolo rispetto alle variabili i-a-S. II vertice insegnante non è di pertinenza solo del docente, ma può essere assunto da altri individui/account. In particolari configurazioni del triangolo, non tutti gli allievi di una classe appartengono al vertice allievo, ma possono distribuirsi tra il vertice allievo e il vertice insegnante e lo stesso docente si potrebbe trovare nel ruolo di allievo per quanto concerne, ad esempio, conoscenze relative all'uso delle tecnologie digitali. II vertice sapere non è occupato esclusivamente dal Sapere accademico, ma può accogliere le diverse declinazioni del sapere previste dalle diverse fasi della trasposizione didattica.

Nel primo episodio abbiamo mostrato come nel passaggio dalla classe fisica alla GC il Triangolo evolve da una situazione in cui i vertici i-a-S sono assunti rispettivamente dalla docente di matematica, dagli alunni della classe e dal Sapere accademico a quella in cui tale configurazione viene traslata in GC e successivamente il vertice insegnante si istanzia in $\mathrm{S}_{7}$, quello di allievo negli altri studenti della classe che partecipano alla discussione e il Sapere è il sapere appreso da $S_{7}$ dal quale prende avvio la trasposizione didattica.

Nel secondo esempio, la complessità dell'evoluzione dei processi di I/A è rappresentata dalla Figura 7. Le variabili i-a-S della relazione triadica vengono istanziate, nella dinamicità del Triangolo:

- dal docente di matematica curriculare, dal docente delle ripetizioni, dal familiare esperto e da $S_{1}$, per quanto riguarda il vertice insegnante;

- dagli alunni della classe, da $\mathrm{S}_{1}$ e da $\mathrm{S}_{2}$, per quanto concerne il vertice allievo;

- dal Sapere accademico e dal sapere appreso, per quanto concerne il vertice Sapere da cui inizia la trasposizione didattica.

L'altro fattore di natura dinamica che occorre considerare per disegnare l'evoluzione dei processi di I/A, quando classi fisiche e classi virtuali si contaminano, è il tipo di pratiche che caratterizza le società-classi che emergono da tale contaminazione. In particolare, le pratiche funzionali, le meta-pratiche extrafunzionali e la loro reciproca interazione.

Nel primo episodio, per ottemperare all'invito dell'insegnante, avvenuto nella società-classe fisica, di postare nello Stream di GC gli esercizi per casa, si possono ravvisare le dinamiche derivanti dall'interazione tra pratiche funzionali e meta-pratiche 
extrafunzionali. I primi esercizi postati nello Stream mostrano l'interferenza di meta-pratiche della società-classe fisica che vanno a interferire con le pratiche funzionali costitutive della società-GC. Infatti, i primi studenti mostrano una certa ritrosia ad esporsi mostrando ai compagni e agli insegnanti gli esercizi svolti sul proprio quaderno e si limitano a indicare quali esercizi non sono riusciti a svolgere. Una meta-pratica, mutuata dalla società-classe fisica e riconducibile al contratto didatti$\mathrm{Co}$, interferisce con la pratica funzionale che consiste nell'apprendimento della matematica. All'interno della società-GC notiamo, tuttavia, un'evoluzione da meta-pratica a pratica funzionale innescata da $\mathrm{S}_{7}$ che nel contesto della classe virtuale si prende la responsabilità di assumere il ruolo di insegnante e di operare una trasposizione da sapere appreso $\left(\mathrm{da}_{7}\right)$ a sapere da insegnare a sapere insegnato a sapere appreso. Nel territorio della società-classe fisica nella quale la relazione triadica i-a-S è più statica, $\mathrm{S}_{7}$ probabilmente non avrebbe trovato in sé e nel gruppo il sostegno affettivo-relazionale di rompere il contratto didattico.

Nel secondo esempio osserviamo uno scivolamento da parte di $S_{2}$ da pratica funzionale a meta-pratica extrafunzionale. In effetti, se $\mathrm{S}_{2}$ inizia l'interazione con $\mathrm{S}_{1}$ attuando una pratica funzionale volta all'apprendimento della matematica - nel caso specifico il bisogno e il desiderio di comprendere l'esercizio sullo studio di funzioni - nell'affrontare il secondo esercizio il suo comportamento è riconducibile a meta-pratiche extrafunzionali descritte nel par. 4.2.2.

In definitiva, la possibilità di dipanare la complessità evidenziata da Borba et al. (2016) che emerge quando le tecnologie digitali incontrano gli spazi di apprendimento fisici, è da attribuire alla forza epistemologica del Triangolo e della nozione di pratica e meta-pratica introdotta da D'Amore in Didattica della Matematica. Queste due lenti teoriche, a nostro avviso, permettono di accogliere la dinamicità e la complessità sistemico-relazionale che emergono dall'interazione di classi fisiche e virtuali.

\section{Conclusione}

Nel presente lavoro abbiamo analizzato due esempi di dinamiche di I/A in contesti arricchiti dall'uso delle tecnologie digitali usando una lente teorica composta dai seguenti elementi:

- lo schema triadico del Triangolo, per inquadrare le particolarizzazioni e le relazioni tra gli attori, la cui dinamicità è tipica dei contesti di l/A in cui sono presenti delle tecnologie digitali (Albano, 2017);

- la prospettiva sociologica di classe intesa come società proposta da D'Amore, usata per mettere in relazione le pratiche e le meta-pratiche delle diverse società-classe in gioco.

Il primo esempio considerato è uno scambio di messaggi nello Stream di GC avvenuto in una seconda Liceo Scientifico. II secondo esempio considerato è un estratto di una chat privata su Whatsapp tra due studenti compagni di classe che frequentano I'ultimo anno di Liceo Classico. Entrambi gli esempi, riguardano l'interazione tra spazi di I/A fisici e virtuali e sono episodi realmente avvenuti che noi abbiamo analizzato a posteriori. Abbiamo voluto proporre un approccio e una prospettiva 
teorica per poter inquadrare e studiare questioni di questo tipo anche quando esse si realizzano nella complessità dei contesti reali. Lo schema triadico del Triangolo ci ha permesso di caratterizzare didatticamente le diverse interazioni tra gli individui. La visione di una classe come società ci ha permesso di approcciare ad una situazione complessa come quella in cui spazi fisici interagiscono con spazi virtuali attraverso una lente analitica che, facendo chiarezza tra territori comuni, individui/ account coinvolti, interazioni e modalità di interazione e comportamenti (guardati in termini di pratiche e meta-pratiche), a nostro avviso aiuta ad operare un'analisi didattica più nitida.

I due esempi, pur essendo tra loro molto diversi, attraverso I'analisi teorica hanno rivelato alcuni punti in comune che siamo riusciti ad individuare e specificare. II primo punto riguarda l'inevitabile contaminazione tra ciò che avviene nel contesto della classe fisica e ciò che avviene negli spazi virtuali. Tale contaminazione si è rivelata più forte nel primo esempio, in cui il piano fisico e quello virtuale hanno dato vita ad un nuovo spazio emergente che li vede quasi intrecciati, ma è comunque presente nel secondo, in cui le maglie del contratto didattico della classe fisica riescono ad infiltrarsi fin dentro lo spazio privato di due studenti. Il secondo punto, riguarda il riscontro della presenza di elementi quali devoluzione, implicazione personale, costruzione di conoscenza e rottura del contratto didattico nelle interazioni che avvengono negli spazi virtuali. Naturalmente, questo non significa che tali elementi esistano solamente in tali spazi né che lì esistano sempre ma il punto, a nostro avviso, di estremo interesse è che in tali spazi si vedono, diventano osservabili e studiabili. Un terzo punto, legato al secondo e che accomuna entrambi gli esempi riportati, riguarda la difficoltà di distinguere con nitidezza le pratiche dalle meta-pratiche soprattutto nei contesti ibridi che stiamo considerando. Infatti, la fluidità e la dinamicità che caratterizzano tali contesti non solo aumentano la complessità del sistema studiato ma ne sfumano anche i confini. Con riferimento al già citato Borba et al. (2016) - che mette in evidenza come la tecnologia digitale abbia completamente destrutturato I'immagine degli ambienti di apprendimento tradizionali, abilitando nuove tipologie di classi e di modalità di lavoro che, a loro volta, innescano differenti dinamiche di natura socio-culturale - gli episodi che abbiamo analizzato mostrano come le tecnologie digitali siano state determinanti nel modificare la natura del Triangolo e della Trasposizione didattica. Tuttavia, riteniamo che la ricerca in questo ambito debba continuare approfondendo il ruolo delle tecnologie digitali nel modificare un'altra dimensione fondamentale che contraddistingue l'apprendimento della matematica: ci riferiamo al ricchissimo mondo della semiotica nelle sue declinazioni strutturali funzionali e semiotico-culturali.

Ci sentiamo di concludere questo lavoro non tanto con delle risposte ma con alcune riflessioni di carattere generale e l'esplicitazione di un'esigenza di definire sempre meglio i problemi di ricerca in questo ambito. Le tecnologie digitali in un contesto di l/A agiscono in maniera analoga ad un prisma che rifrange la luce: scompongono soggetti, oggetti e processi didattici, spacchettando e rendendo visibili (e, quindi, analizzabili) le loro varie sottocomponenti. In questo senso, è emblematico anche il secondo esempio da noi considerato: la raccolta e l'archiviazione di dati è implicita quando si utilizzano strumenti come Whatsapp e, grazie alla preziosa disponibilità di $\mathrm{S}_{1}$ ed $\mathrm{S}_{2}$, abbiamo avuto la possibilità di studiare un autentico scambio privato tra studenti senza averlo contaminato con la nostra presenza, sia essa dichiarata o nascosta. Riteniamo che tutto questo abbia, e avrà sempre di più, un impatto notevole a livello di metodologia di ricerca in didattica. 


\section{Ringraziamenti}

Desideriamo ringraziare $S_{1}$ ed $S_{2}$ per il loro coraggio e la loro disponibilità nel mettere a nostra disposizione il contenuto della loro chat privata.

Ringraziamo caldamente Bruno D'Amore e Miglena Asenova per le profonde osservazioni e i suggerimenti che hanno contribuito ad un sensibile miglioramento della versione iniziale dell'articolo.

Ringraziamo infine i due anonimi referee per i loro preziosi commenti che sono stati determinanti per arrivare alla forma definitiva del lavoro.

Questo articolo è uno dei risultati del progetto di ricerca VIRMATH della Libera Università di Bolzano, Facoltà di Scienze della Formazione.

\section{Bibliografia}

Albano, G. (2017). e-Mathematics Engineering for Effective Learning. In G. Aldon, F. Hitt, L. Bazzini \& U. Gellert (Eds.), Mathematics and Technology (pp. 349-370). Springer International Publishing.

Albano, G., Faggiano, E., \& Mammana, M. F. (2013). A tetrahedron to model e-learning Mathematics. Quaderni di Ricerca in Didattica (Mathematics), 23 (Supplemento 1), 429436, G.R.I.M. (Department of Mathematics and Computer Science, University of Palermo, Italy).

Bagni, G. T., \& D'Amore, B. (2005). Epistemologia, sociologia, semiotica: la prospettiva socio-culturale. La matematica e la sua didattica, 1, 73-89.

Borba, M. C., Askar, P., Engelbrecht, J., Gadanidis, G., Llinares, S., \& Aguilar, M. S. (2016). Blended learning, e-learning and mobile learning in mathematics education. ZDM, 48(5), 589-610.

Brousseau, G. (1986). Fondements et méthodes de la didactique des mathématiques Recherches en Didactique des Mathématiques, 7(2), 33-115.

Brousseau, G. (2002). Theory of didactical situations in mathematics: Didactique des mathématiques, 1970-1990. New York/Boston/Dordrecht/London/Moscow: Kluwer Academic Publishers.

Chevallard, Y., \& Joshua, M. A. (1982). Un exemple d'analyse de la transposition didactique: la notion de distance. Recherches en didactique des mathématiques, 3(1), 159-239.

D'Amore, B. (1999). Elementi di didattica della matematica. Bologna: Pitagora

D'Amore, B. (2003). Le basi filosofiche, pedagogiche, epistemologiche e concettuali della didattica della matematica. Bologna: Pitagora.

D'Amore, B. (2005). Pratiche e metapratiche nell'attività matematica della classe intesa come società. Alcuni elementi rilevanti della didattica della matematica interpretati in chiave sociologica. La matematica e la sua didattica, 3, 325-336.

D'Amore, B., \& Fandiño Pinilla, M. I. (2002). Un acercamiento analitico al "triángulo de la didáctica". Educación Matemática (México DF, México), 14(1), 48-61.

Del Zozzo, A. (2019). VirMath. Classi virtuali in matematica. Report interno dei primi risul- 
Prospettive teoriche per lo studio della contaminazione tra ambienti di insegnamento/apprendimento fisici e virtuali / Agnese Del Zozzo e George Santi

tati del progetto VirMath della Facoltà di Scienze della Formazioni della Libera Università di Bolzano con la supervisione del Professor Giorgio Bolondi. Versione settembre 2019.

Del Zozzo, A., \& Santi, G. (2019). Physical and virtual classroom in the learning of mathematics: analysis of two episodes (poster). In U. T. Jankvist, M. Van den Heuvel-Panhuizen \& M. Veldhuis (Eds.), Proceedings of the Eleventh Congress of the European Society for Research in Mathematics Education CERME11, February 6 - 10, 2019. Utrecht, the Netherlands: Freudenthal Group \& Freudenthal Institute, Utrecht University and ERME.

Drijvers, P. H. M., Ball, L., Barzel, B., Heid, M. K., Cao, Y., \& Maschietto, M. (2016). Uses of technology in lower secondary mathematics education: A concise topical survey. NY: Springer.

Fandiño Pinilla, M. I. (2002). Curricolo e valutazione. Bologna: Pitagora.

Fandiño Pinilla, M. I. (2003). "Diventare competente", una sfida con radici antropologiche. In B. D’Amore, J. D. Godino, G. Arrigo, \& M. I. Fandiño Pinilla (Eds.), Competenze in matematica (pp. 75-88). Bologna: Pitagora.

Merton, R. (1968). Social Theory and Social Structure. New York: Free Press.

Risk, U. (2002). Draft standard for learning object metadata. IEEE Standard, 1484(1).

Sfard, A. (2008). Psicologia del pensiero matematico. Trento: Erickson.

\author{
Autori/Agnese Del Zozzo e George Santi \\ Facoltà di Scienze della Formazione, Libera Università di Bolzano - Italia; \\ Nucleo di Ricerca Didattica della matematica, Università di Bologna - Italia \\ adelzozzo@unibz.it, gesanti@unibz.it
}

